\title{
Schur-geometric and Schur-harmonic Convexity of an Integral Mean for Convex Functions
}

\author{
Jian Sun ${ }^{1}$, Zhi-Ling Sun ${ }^{1}$, Bo-Yan $X^{1}{ }^{1}$, Feng $Q^{i^{2,3, *}}$ \\ ${ }^{1}$ College of Mathematics, Inner Mongolia University for Nationalities, Tongliao City, Inner Mongolia Autonomous Region, China \\ ${ }^{2}$ Department of Mathematics, School of Science, Tianjin Polytechnic University, Tianjin City, China \\ ${ }^{3}$ Institute of Mathematics, Henan Polytechnic University, Jiaozuo City, Henan Province, China \\ *Corresponding author: qifeng618@gmail.com
}

Received July 12, 2015; Revised August 15, 2015; Accepted August 27, 2015

\begin{abstract}
In the paper, Schur-geometric and Schur-harmonic convexity of an integral mean for convex functions are established.
\end{abstract}

Keywords: Schur-convex function, Schur-geometrically convex function, Schur-harmonically convex function, inequality, generalized logarithmic mean

Cite This Article: Jian Sun, Zhi-Ling Sun, Bo-Yan Xi, and Feng Qi, "Schur-geometric and Schur-harmonic Convexity of an Integral Mean for Convex Functions." Turkish Journal of Analysis and Number Theory, vol. 3, no. 3 (2015): 87-89. doi: 10.12691/tjant-3-3-4.

\section{Introduction}

In [3], N. Elezović and J. Pečarić established the following theorem.

Theorem A ([3]). Let $f: I \subseteq R \rightarrow R$ and $a, b \in I^{\circ}$. Then

$$
F(a, b)= \begin{cases}\frac{1}{b-a} \int_{a}^{b} f(x) \mathrm{d} x, & a \neq b, \\ f(a), & a=b\end{cases}
$$

is Schur-convex (Schur-concave) on $I^{2}$ if and only if $f$ is convex (concave) on $I$.

In $[7,10]$, Theorem A was generalized as the following theorem.

Theorem B $([7,10])$. Let $f$ be a continuous function and $p$ a positive continuous weight on an interval $I$. Then the weighted arithmetic mean of $f$ with weight $p$ defined by

$$
G(x, y)= \begin{cases}\frac{\int_{x}^{y} p(t) f(t) \mathrm{d} t}{\int_{x}^{y} p(t) \mathrm{d} t}, & x \neq y, \\ f(x), & x=y\end{cases}
$$

is Schur-convex (Schur-concave) on $I^{2}$ if and only if

$$
\frac{\int_{x}^{y} p(t) f(t) \mathrm{d} t}{\int_{x}^{y} p(t) \mathrm{d} t} \leq \frac{p(x) f(x)+p(y) f(y)}{p(x)+p(y)}
$$

holds (reverses) for all $(x, y) \in I^{2}$.

For more information on this topic, please refer to $[5,8,9]$ and closely-related references therein.
In this paper, we discuss Schur-geometric and Schurharmonic convexity of the mean $F(a, b)$ and obtain two results which generate Theorem A.

\section{Definitions and Lemmas}

In order to prove our main results we need the following definitions and lemmas.

Definition 1 ([4]). Let $I \subseteq R$ and $x=\left(x_{1}, \cdots, x_{n}\right)$, $y=\left(y_{1}, \cdots, y_{n}\right) \in I^{n}$, and let $\varphi: I^{n} \rightarrow R$.

(1) $x$ is said to be majorized by $y$ (in symbols $x \prec y$ ) if $\quad \sum_{i=1}^{k} x_{[i]} \leq \sum_{i=1}^{k} y_{[i]} \quad$ for $\quad k=1,2, \cdots, n-1 \quad$ and $\sum_{i=1}^{n} x_{[\mathrm{i}]}=\sum_{i=1}^{n} y_{[i]} \quad, \quad$ where $\quad x_{[1]} \geq \cdots \geq x_{[n]} \quad$ and $y_{[1]} \geq \cdots \geq y_{[n]}$ are rearrangements of $x$ and $y$ in a descending order.

(2) $x \leq y$ means $x_{i} \leq y_{i}$ for all $i=1,2, \cdots, n . \phi$ is said to be increasing if $x \leq y$ implies $\varphi(x) \leq \varphi(y)$. $\phi$ is said to be decreasing if and only $-\phi$ is increasing.

(3) $\phi$ is said to be a Schur-convex function on $I^{n}$ if $x \prec y$ on $I^{n}$ implies $\varphi(x) \leq \varphi(y)$. $\phi$ is said to be a Schur-concave function on $I^{n}$ if and only $-\phi$ is Schurconvex function.

Definition $2([1,2])$. Let $x=\left(x_{1}, \cdots, x_{n}\right), y=\left(y_{1}, \cdots, y_{n}\right)$ $\in I^{n} \subseteq R_{+}^{n}$ and $\varphi: I^{n} \rightarrow R$ and let $\ln x=\left(\ln x_{1}, \cdots, \ln x_{n}\right)$, $\frac{1}{x}=\left(\frac{1}{x_{1}}, \cdots, \frac{1}{x_{n}}\right)$.

(1) $\phi$ is said to be a Schur-geometrically convex function on $I^{n}$ if $\ln x \prec \ln y$ on $I^{n}$ implies $\varphi(x) \leq \varphi(y)$. 
$\phi$ is said to be a Schur-geometrically concave function on $I^{n}$ if and only $-\phi$ is Schur-geometrically convex function.

(2) $\phi$ is said to be a Schur-harmonically convex function on $I^{n}$ if $\frac{1}{x} \prec \frac{1}{y}$ on $I^{n}$ implies $\varphi(x) \leq \varphi(y) \cdot \phi$ is said to be a Schur-harmonically concave function on $I^{n}$ if and only $-\phi$ is Schur-harmonically convex function.

Lemma 2.1([1]). Let $\varphi: I^{2} \subseteq R_{+}^{2} \rightarrow R$ be a continuous function on $I^{2}$ and differentiable in interior of $I^{2}$. Then $\varphi$ is Schur-geometrically convex (Schur-geometrically concave) if and only if it is symmetric and

$$
(b-a)\left(b \frac{\partial \varphi}{\partial b}-a \frac{\partial \varphi}{\partial a}\right) \geq(\leq) 0
$$

for all $a, b \in I$.

Lemma 2.2 ([2]). Let $\varphi: I^{2} \subseteq R_{+}^{2} \rightarrow R$ be a continuous function on $I^{2}$ and differentiable in interior of $I^{2}$. Then $\varphi$ is Schur-harmonically convex (Schurharmonically concave) if and only if it is symmetric and

$$
(b-a)\left(b^{2} \frac{\partial \varphi}{\partial b}-a^{2} \frac{\partial \varphi}{\partial a}\right) \geq(\leq) 0
$$

for all $a, b \in I$.

For two positive numbers $a>0$ and $b>0$, define

$$
\begin{aligned}
& A(a, b)=\frac{a+b}{2}, \\
& G(a, b)=\sqrt{a b}, \\
& H(a, b)=\frac{2 a b}{a+b}
\end{aligned}
$$

and

$$
L_{r}(a, b)= \begin{cases}\left(\frac{b^{r+1}-a^{r+1}}{(r+1)(b-a)}\right)^{1 / r}, & a \neq b, r \geq 1, \\ a, & a=b .\end{cases}
$$

It is well known that $A(a, b), G(a, b), H(a, b)$ and $L_{S}(a, b)$ are respectively called the arithmetic, geometric, harmonic and generalized logarithmic means of $a$ and $b$.

Lemma 2.3 ([6]) $L_{r}(a, b)$ is increasing function on $(a, b) \in R_{+}^{2}$.

In this paper, we will prove that the function $F(a, b)$ is Schur-geometrically convex and Schur-harmonically convex on $R_{+}^{2}$.

\section{Main Results}

Theorem 3.1. Let $f: I \subseteq R_{+} \rightarrow R$ and $F$ be defined in Theorem A.

(i). If $f$ is convex and increasing on $I$, then $F$ is Schur-geometrically convex on $I^{2}$. (ii). If $f$ is concave and decreasing on $I$, then $F$ is Schur-geometrically concave on $I^{2}$.

Proof. If $a, b \in I^{\circ}$ and $a=b$, we have $F(a, a)=f(a)$.

For all $a, b \in I^{\circ}, a \neq b$, a straightforward computation gives

$$
\begin{aligned}
& \frac{\partial F}{\partial b}=\frac{1}{b-a} f(b)-\frac{1}{b-a} F(a, b), \\
& \frac{\partial F}{\partial a}=-\frac{1}{b-a} f(a)+\frac{1}{b-a} F(a, b) .
\end{aligned}
$$

If $f$ is convex and increasing on $I$, by the inequality (2), we obtain

$$
\begin{aligned}
& (b-a)\left(b \frac{\partial F}{\partial b}-a \frac{\partial F}{\partial a}\right) \\
= & a f(a)+b f(b)-(a+b) F(a, b) \\
= & a f(a)+b f(b)-\frac{a+b}{b-a} \int_{a}^{b} f(x) \mathrm{d} x \\
\geq & \frac{1}{2}[2 a f(a)+2 b f(b)-(a+b)(f(a)+f(b))] \\
= & \frac{1}{2}(b-a)(f(b)-f(a)) \geq 0 .
\end{aligned}
$$

Hence, $F(a, b)$ is Schur-geometrically convex on $I^{2}$. If $f$ is concave and decreasing on $I$, then the inequality (4) is reversed. According to Lemma 2.1, it follows that $F(a, b)$ is Schur-geometrically concave $I^{2}$. This completes the proof of Theorem 3.1 .

Theorem 3.2. Let $f: I \subseteq R_{+} \rightarrow R$ and $F$ be defined in Theorem A.

(i). If $f$ is convex and increasing on $I$, then $F$ is Schur-harmonically convex on $I^{2}$.

(ii). If $f$ is concave and decreasing on $I$, then $F$ is Schur-harmonically concave on $I^{2}$.

Proof. If $a, b \in I^{\circ}$ and $a=b$, we have $F(a, a)=f(a)$. For all $a, b \in I^{\circ}, a \neq b$, if $f$ is convex and increasing, using inequality (3) and (2), we get

$$
\begin{aligned}
& (b-a)\left(b^{2} \frac{\partial F}{\partial b}-a^{2} \frac{\partial F}{\partial a}\right) \\
= & a^{2} f(a)+b^{2} f(b)-\frac{a^{2}+b^{2}}{b-a} \int_{a}^{b} f(x) \mathrm{d} x \\
\geq & a^{2} f(a)+b^{2} f(b)-\frac{a^{2}+b^{2}}{2}(f(a)+f(b)) \\
= & \frac{1}{2}\left(b^{2}-a^{2}\right)(f(b)-f(a)) \geq 0 .
\end{aligned}
$$

Therefore, $F(a, b)$ is Schur-harmonically convex function on $I^{2}$. If $f$ is concave and decreasing function on $I$, then the inequality (5) is reversed. According to Lemma 2.2 , it follows that $F(a, b)$ is Schur-harmonically concave function on $I^{2}$. The proof of Theorem 3.2 is complete. 


\section{Applications}

Theorem 4.1. For $a>0$ and $b>0$, if $r \geq 1$, then $L_{r}(a, b)$ is Schur-geometrically convex and Schurharmonically convex.

Proof. Taking $f(x)=x^{r}$ for all $x \in R_{+}$, if $a \neq b$, it follows that

$F(a, b)=\frac{1}{b-a} \int_{a}^{b} f(x) \mathrm{d} x=\frac{1}{b-a} \int_{a}^{b} x^{r} \mathrm{~d} x=\frac{b^{r+1}-a^{r+1}}{(r+1)(b-a)}$

and $f(x)=x^{r}$ is convex increasing on $R_{+}$for $r \geq 1$. Therefore, by Theorem 3.1 and 3.2, we have

$$
F(a, b)= \begin{cases}\frac{b^{r+1}-a^{r+1}}{(r+1)(b-a)}, & a \neq b, \\ a^{r}, & a=b\end{cases}
$$

is Schur-geometrically convex and Schur-harmonically convex on $R_{+}^{2}$ for $r \geq 1$, then $L_{r}(a, b)$ is Schurgeometrically convex and Schur-harmonically convex on $R_{+}^{2}$ for $r \geq 1$. Thus, Theorem 4.1 is proved.

Corollary 4.1.1. For $b>a>0$ and $r \geq 1$, define $u_{a}=t a+(1-t) b \quad, \quad v_{a}=(1-t) a+t b \quad, \quad u_{g}=a^{t} b^{1-t}$, $v_{g}=a^{1-t} b^{t} \quad, \quad u_{h}=t a^{-1}+(1-t) b^{-1}, \quad$ and $v_{h}=(1-t) a^{-1}+t b^{-1}$ for $t \in(0,1)$. Then

(1) when $t \in(0,1)$ and $t \neq 1 / 2$, we have

$$
\begin{aligned}
& {\left[\frac{u_{h}^{-(r+1)}-v_{h}^{-(r+1)}}{(r+1)\left(u_{h}^{-1}-v_{h}^{-1}\right)}\right]^{1 / r} \leq\left[\frac{u_{g}^{r+1}-v_{g}^{r+1}}{(r+1)\left(u_{g}-v_{g}\right)}\right]^{1 / r}} \\
& \leq\left[\frac{u_{a}^{r+1}-v_{a}^{r+1}}{(r+1)\left(u_{a}-v_{a}\right)}\right]^{1 / r} \leq L_{r}(a, b)
\end{aligned}
$$

(2) when $t=1 / 2$, we have

$$
H(a, b) \leq G(a, b) \leq A(a, b)=L_{r}(a, b) .
$$

Proof. When $t=1 / 2$, it is easy to obtain that $H(a, b) \leq G(a, b) \leq L_{r}(a, b)$. When $t \in(0,1)$ and $t \neq 1 / 2$, by Corollary 2 in [6] and Lemma 2.3, Corollary 4.1 .1 is thus proved.

\section{Acknowledgements}

The authors thank the anonymous referees for their careful corrections to and valuable comments on the original version of this paper.

\section{Support}

This work was partially supported by the National Natural Science Foundation of China under Grant No. 11361038 and by the Inner Mongolia Autonomous Region Natural Science Foundation Project under Grant No. 2015MS0123 and No. 2014BS0106, China.

\section{References}

[1] Y.-M. Chu, X.-M. Zhang, and G.-D. Wang, The Schur geometrical convexity of the extended mean values, J. Convex Anal. 15 (2008), no. 4, 707-718.

[2] W.-F. Xia and Y.-M. Chu, Schur-convexity for a class of symmetric functions and its applications, J. Inequal. Appl. 2009 (2009), Article ID 493759, 15 pages.

[3] N. Elezović and J. Pečarić, A note on Schur-convex functions, Rocky Mountain J. Math. 30 (2000), no. 3, 853-856.

[4] A. W. Marshall and I. Olkin, Inequalities: Theory of Majorization and its Application, New York: Academies Press, 2011.

[5] C. Mortici, Arithmetic mean of values and value at mean of arguments for convex functions, ANZIAM J. 50 (2008), no. 1, 137-141.

[6] F. Qi and Q.-M. Luo, A simple proof of monotonicity for extended mean values, J. Math. Anal. Appl. 224 (1998), 356-359.

[7] F. Qi, J. Sándor, S. S. Dragomir, and A. Sofo, Notes on the Schurconvexity of the extended mean values, Taiwanese J. Math. 9 (2005), no. 3, 411-420.

[8] H.-N. Shi, Schur-convex functions related to Hadamard-type inequalities, J. Math. Inequal. 1 (2007), no. 1 127-136.

[9] H.-N. Shi, D.-M. Li and C. Gu, The Schur-convexity of the mean of a convex function, Appl. Math. Lett. 22 (2009), no. 6, 932-937.

[10] H.-N. Shi, S.-H. Wu, and F. Qi, An alternative note on the Schurconvexity of the extended mean values, Math. Inequal. Appl. 9 (2006), no. 2, 219-224. 